\title{
A Non-seed-based Region Growing Algorithm for High Resolution Remote Sensing Image Segmentation
}

\author{
Lin $\mathrm{Wu}^{1(\bowtie)}$, Yunhong Wang ${ }^{2}$, Jiangtao Long ${ }^{1}$, and Zhisheng Liu ${ }^{1}$ \\ ${ }^{1}$ Chongqing Communication College, Chongqing 400035, China \\ wulin@buaa.edu.cn \\ 2 Beihang University, Beijing 100191, China \\ yhwang@buaa.edu.cn
}

\begin{abstract}
One of the indispensable prerequisites for high resolution remote sensing image interpretation and processing is successful image segmentation. The algorithm presented in this paper aims for a high efficient image segmentation applicable and adaptable to high resolution remote sensing images. This is achieved by a non-seed-based region growing, which constructs neighbor pairwise pixel stack instead of depending on any seed points. The stack is constructed in increasing order of neighbor pairwise pixel spectral difference which is computed based on 4-connexity. The proposed algorithm carries out region growing according to the merging criterion (i.e. grow formula) and traversal of the stack. We apply the proposed and conventional region growing algorithms to two data sets of ZiYuan-3 (ZY-3) high resolution remote sensing images and analyze the segmentation results based on Carleer evaluation method that manifests high efficient segmentation of the proposed algorithm.
\end{abstract}

Keywords: High resolution remote sensing image - Image segmentation Non-seed-based region growing $\cdot$ Ziyuan-3 (ZY-3) $\cdot$ Carleer evaluation method

\section{Introduction}

Till date, with the emergence of a host of high resolution remote sensing satellites (such as IKONOS, GeoEye-1, QuickBird, WorldView-2, ZY-3, GF-1, etc.), substantial high resolution remote sensing images are increasingly applied in the field of environmental protection, land survey, disaster assessment, military target monitoring, etc. Compared with low or moderate resolution remote sensing images, high resolution remote sensing images contain more detailed information, such as shape, texture, etc.; however, the noise is more pronounced. Nowadays, object-oriented image processing techniques have received great attention and demonstrate more advantages in remote sensing change detection, object detection, etc. An indispensable step for object-oriented image processing is image segmentation. Remote sensing image segmentation is a process of dividing it into non-overlapping areas in space, the result is a set of interconnected, considerable homogeneity surface features image regions [1].

A large variety of image segmentation algorithms were developed during the last 20 years. Due to the complexity of the high resolution remote sensing image scene, two 
main groups of image segmentation algorithms (boundary-based and region-based) are widely used for high resolution remote sensing images. The boundary-based algorithms detect object contours explicitly using the discontinuity property, and the region-based algorithms locate image regions explicitly according to the similarity property [2]. The region-based algorithms are less sensitive to texture and noise which is a significant advantage in high resolution remote sensing image segmentation and the implementation of multi-level segmentation is easier with region-based technique as long as the heterogeneity tolerance is increased, consequently, region-based algorithms will be the mainstream in the foreseeable future [3].

A quintessential region-based algorithm is region growing which was first introduced by Rolf Adams and Leanne Bischof in 1994 [4]. It starts with initial seed points and grows with neighboring homogenous regions, then in numerous subsequent steps, smaller image regions are merged into bigger ones, if the two neighbor image regions satisfy the merging criterion. Seed points may be one pixel or region. If the smallest growth exceeds a heterogeneity tolerance defined by the user, the merging process stops [5]. The heterogeneity tolerance affects the relative size of output image regions. Nowadays, region growing is widely used for high resolution remote sensing image segmentation. At first, Siebert proposed a novel segmentation algorithm based on region growing, in which parameters were dynamically derived from the data for each region based on a local quality measure of the region's contour [6]. Then Chen et al. further proposed a multi-scale image segmentation algorithm combined with multicharacters based on region growing [7]. Afterwards, $\mathrm{Xu}$ et al. proposed an improved segmentation of remote sensing images based on watershed and region growing to reduce the over-segmentation of watershed transform [8]. More recent studies by Li et al. demonstrated that multi-scale segmentation method based on region growing was suitable for high resolution remote sensing image segmentation and particle swarm optimization algorithm was applied to select near-optimal multi-scale segmentation parameters [9].

Technically, in seed based region growing algorithms, selection of initial seed points is crucial because it extremely decides the accuracy of segmentation by region growing [10]. In recent years, how to select more appropriate seed points for region growing exerts a tremendous fascination on a host of researchers. Cui et al. adopted the Harris corner detector to calculate initial seed points for region growing [11]. Tang utilized watershed algorithm to get over-segmentation image regions and then the centroid of each region was used as initial seed points [12]. Preetha et al. proposed an automatic seeded region growing algorithm, in which seed points were selected based on the similarity and the Euclidean distance of a pixel to its neighbors [13]. Mirghasem et al. proposed a new image segmentation algorithm based on modified seeded region growing, in which seed points were selected based on particle swarm optimization algorithm [14]. But instead seed point selection is affected by particular technique limitation and increases the computation overhead; consequently it is still a challenge for researchers.

The goal of this paper is to propose a non-seed-based region growing algorithm for high resolution remote sensing images. In the proposed algorithm, we firstly implement grow formula preliminary definition and segmentation parameter initialization. Then we construct neighbor pairwise pixel stack in increasing order of neighbor pairwise 
pixel spectral difference. Afterwards, region growing is done by integrating traversal of the stack in order and grow formula which follows the stopping criteria to stop the growth of region. We utilize two ZY-3 high resolution remote sensing images which provide an empirical basis for research on image segmentation.

The remainder of this paper is organized as follows. Section 2 provides a brief review on related work. The proposed algorithm has been discussed in Sect. 3 and in Sect. 4 the experimental results and analysis have been revealed. Finally, this paper is concluded in Sect. 5.

\section{A Brief Review on Related Work}

\subsection{Region Growing}

Region growing algorithms are used to determine image regions directly and cluster pixels starting on a limited number of single seed points. Initial seed points may be a single pixel or a group of pixels called regions. Region growing algorithms basically depend on a set of given seed points, often suffering from a lack of control in the merging criterion for the growth of a region, and then segmentation is done until all the pixels are grouped in any one of the regions. Owing to the impact of noise, high resolution remote sensing image segmentation results invariably exist oversegmentation; consequently region growing algorithms probably require particular region merging steps as a post segmentation procedure.

In [15], a local mutual best fitting heuristics is proposed (see Fig. 1) and has been widely utilized for region growing $[5,7,16]$. In Fig. 1, each polygon is an image region and the procedure of the local mutual best fitting heuristics is as follows: starting with an arbitrary image region $A$, find its neighbor region $B$ with which the merging criterion is fulfilled best. Find for $B$ its neighbor region $C$ with which $B$ fulfills the merging criterion best. Confirm that the merging criterion is best fulfilled mutually (i.e. $C=A$ ). If it is, merge $A$ and $B$. If not, repeat the same loop taking $B$ for $A$ and $C$ for $B$. This heuristic allows to find the best fitting pair of regions in the local vicinity of $A$ following the merging criterion.

\subsection{Evaluation of Segmentation Results}

An army of image segmentation evaluation methods have been proposed over the last several decades. These evaluation methods are roughly divided into unsupervised and supervised methods, based on whether the method requires a ground-truth reference image or not [17]. Supervised evaluation methods [3, 18, 19], also known as empirical discrepancy methods [20] or relative evaluation methods [21], evaluate image segmentation algorithms by comparing the resulting segmented image against a manually-segmented reference image, which is often referred to ground-truth. The degree of similarity between the human and machine segmented images determines the quality of the segmented image. Contrary to supervised evaluation methods, unsupervised evaluation methods [17], also known as empirical goodness methods [20] or stand-alone evaluation methods [22], do not require a reference image, but instead 


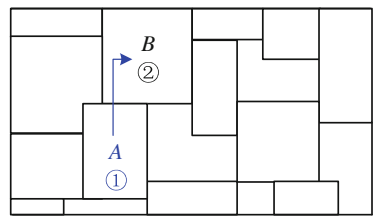

(a) starting with object $A$, find its neighbor object $B$ with which the merging criterion is fulfilled best

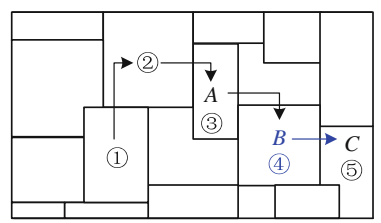

(d) taking $B$ for $A$ and $C$ for $B$, repeat the same loop in (b), but $C \neq A$

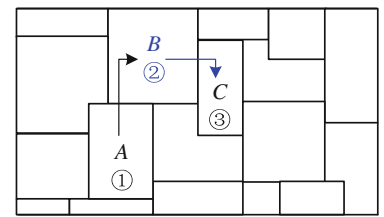

(b) find for $B$ its neighbor object $C$ with which $B$ fulfills the merging criterion best, but $C \neq A$

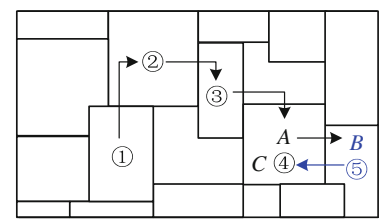

(e) taking $B$ for $A$ and $C$ for $B$, repeat the same loop in (b), $C=A$

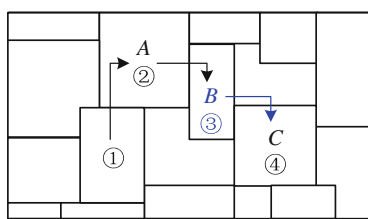

(c) taking $B$ for $A$ and $C$ for $B$, repeat the same loop in (b), but $C \neq A$

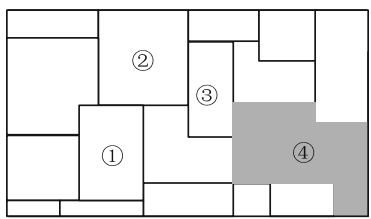

(f) merge $A$ and $B$

Fig. 1. Local mutual best fitting heuristics

evaluate a segmented image based on how well it matches a host of characteristics of segmented images as desired by humans.

Although manually generating a reference image is an arduous, subjective, and time-consuming task, one potential benefit of supervised evaluation methods over unsupervised evaluation methods is that the direct comparison between a segmented image and a reference image is believed to provide a finer evaluation [17]. For supervised evaluation methods, a large variety of discrepancy measures have been proposed. Most early methods evaluated segmented images based on the number of mis-classified pixels versus the reference image [23, 24]. Another group of discrepancy measures are based on the differences in the feature values measured from the segmented images and the reference image $[25,26]$. These methods have been extended to accommodate the problem when the image region number differs between the segmented and reference images [3, 27].

Single discrepancy measure is arduous to fully evaluate the segmentation quality, consequently, a combination of two or more discrepancy measures are often utilized in practice. In this paper, we utilize Carleer evaluation method [3] which comprises two discrepancy measures for evaluating segmentation quality. The first discrepancy measure, based on the number of mis-segmented pixels in the segmented images compared with the manually-segmented reference images (see Fig. 2). The second discrepancy measure is a simple ratio of region number between the segmented and reference images. The two discrepancy measures are defined as follows:

$$
\begin{gathered}
E=\frac{N_{\text {mis }}}{N_{\text {total }}} \times 100 \% \\
G=\frac{N_{a c t}}{N_{\text {ref }}}
\end{gathered}
$$


where $E$ is the percentage of mis-classified pixels, $N_{\text {mis }}$ is the number of mis-classified pixels and $N_{\text {total }}$ is the total number of pixels in the segmented image; $G$ is the ratio of region number, $N_{a c t}$ is the region number in the segmented image and $N_{r e f}$ is the region number in the reference segmentation. This measure allows for evaluation of the over-segmentation $(G>1)$ or the under-segmentation $(G<1)$.

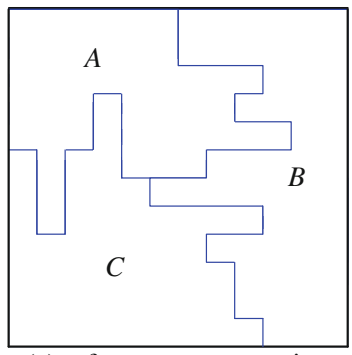

(a) reference segmentation

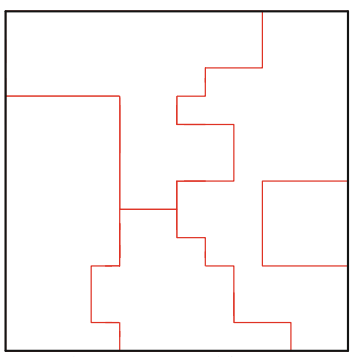

(b) segmented image

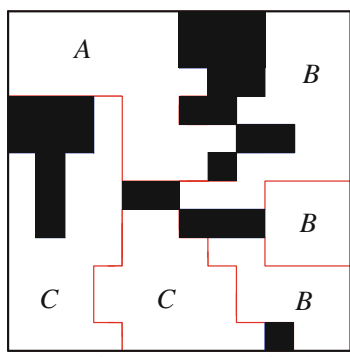

(c) mis-segmented pixels

Fig. 2. Intersection between reference segmentation and segmented image to identify the mis-segmented pixels

It is obviously that without varying $E$, the shorter the distance between $G$ and 1 , the better the segmentation quality. In practice, the combination of above two discrepancy measures, the evaluation can be performed better on the segmentation result, in other words, on the premise of $G$ is approximately equal to 1 , to find the minimum $E$.

\section{The Proposed Approach}

\subsection{Design Goals}

Based on the characteristics of high resolution remote sensing images and conventional region growing algorithms, this section develops a non-seed-based region growing algorithm for high resolution remote sensing image segmentation. It tries to segment the given image by region growing without any seed points and enhance segmentation efficiency on the premise of without debasing segmentation quality. The resulting image regions (also called objects) obtained by the proposed algorithm are the raw material for further classification and refinement procedures. To sum up, the following goals need to be achieved.

Non-seed-based. Due to the deficiency of seed point selection in conventional region growing algorithms, the proposed algorithm would be seed-independent and no longer suffer from the negative impact of segmentation accuracy may be caused by seed point selection.

High Efficiency. Conventional region growing algorithms require initial seed point selection, which increases computational cost and execution time. Compared with low or moderate resolution remote sensing images, data bulk of high resolution remote sensing images is greater; consequently the high efficiency segmentation even on large image data sets is indispensable. 


\subsection{Key Steps}

Preliminary Definition and Initialization. The proposed region growing algorithm is dominated by merging criterion which is defined as follows:

$$
\operatorname{Merging}\left(R_{1}, R_{2}\right)=\left\{\begin{array}{l}
\text { true, } \text { if } f_{\text {heter }}<T \\
\text { false, } \text { otherwise }
\end{array}\right.
$$

where $\operatorname{Merging}\left(R_{1}, R_{2}\right)$ is used to determine whether two neighbor regions can be merged; $f_{\text {heter }}$ is the heterogeneity of newly generated region which is a merger of neighbor regions $R_{1}$ and $R_{2} ; T$ is the heterogeneity threshold (also called segmentation scale). The heterogeneity which can be expressed in Eq. 4 demonstrates differences between two neighbor regions and is comprised of both shape heterogeneity and spectral heterogeneity.

$$
f_{\text {heter }}=w_{\text {spectrum }} h_{\text {spectrum }}+\left(1-w_{\text {spectrum }}\right) h_{\text {shape }}
$$

where $w_{\text {spectrum }}$ is the weight of spectral heterogeneity and $0 \leq w_{\text {spectrum }} \leq 1 ; h_{\text {spectrum }}$ and $h_{\text {shape }}$ are spectral heterogeneity and shape heterogeneity, respectively. They can be further expressed as follows:

$$
\begin{aligned}
h_{\text {spectrum }} & =\sum_{k} N \sigma_{k}-\left(N_{1} \sigma_{k 1}+N_{2} \sigma_{k 2}\right) \\
h_{\text {shape }} & =N \frac{N}{M}-\left(N_{1} \frac{N_{1}}{M_{1}}+N_{2} \frac{N_{2}}{M_{2}}\right)
\end{aligned}
$$

where $N$ and $\sigma_{k}$ are pixel number and standard deviation of newly generated region in the $k$ th spectral band. $N_{1}, N_{2}$ and $\sigma_{k 1}, \sigma_{k 2}$ are pixel number and standard deviation of image regions $R_{1}$ and $R_{2}$ in the $k$ th spectral band, respectively. $M$ is the external rectangular area of newly generated region. $M_{1}$ and $M_{2}$ are external rectangular area of image regions $R_{1}$ and $R_{2}$, respectively.

Through the above analysis, two segmentation parameters should be firstly initialized, i.e. $T$ and $w_{\text {spectrum }}$. The scale is a measure of the maximum size of regions in segmented images, the larger the scale value, the bigger the segments.

Construct Neighbor Pairwise Pixel Stack. As mentioned earlier, the proposed algorithm is non-seed-based, consequently, instead of selecting seed points we compute neighbor pairwise pixel spectral difference between neighbor pixels based on 4-connexity (see Fig. 3a) according to Eq. 7, and then construct neighbor pairwise pixel stack in increasing order of spectral difference according to Eq. 8.

$$
S\left(P_{i}, P_{j}\right)=\sum_{k} \sqrt{\left(L_{k i}-L_{k j}\right)^{2}}
$$




$$
C=\left\{\left(S_{k}, P_{k 1}, P_{k 2}\right) \mid \forall S_{i} \geq S_{j}, \text { if } i \leq j ; 1 \leq i, j, k \leq 2 \times H \times W-H-W\right\}
$$

In Eq. 7, $P_{i}$ and $P_{j}$ are neighbor pixels based on 4-connexity; $S\left(P_{i}, P_{j}\right)$ is spectral difference between $P_{i}$ and $P_{j} ; L_{k i}$ and $L_{k j}$ are the spectral values of $P_{i}$ and $P_{j}$ in the $k$ th spectral band.

In Eq. 8, $P_{k 1}$ and $P_{k 2}$ are neighbor pixels based on 4-connexity; $S_{k}$ is the spectral difference between $P_{k 1}$ and $P_{k 2} ; C$ is the neighbor pairwise pixel stack (see Fig. 3b);

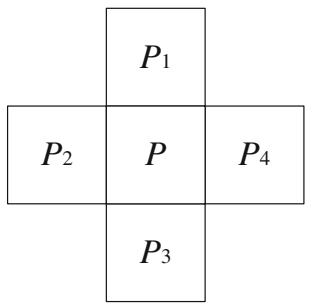

(a)

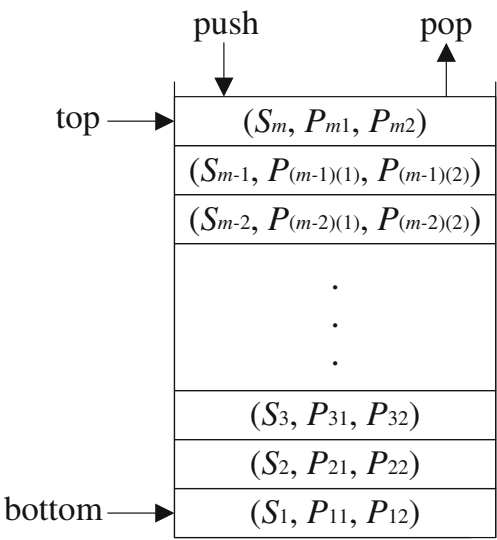

(b)

Fig. 3. The diagrams of 4-connexity and neighbor pairwise pixel stack

$H$ and $W$ are height and width of the given image respectively.

Region Growing. Traverse the pairwise pixel stack $C$, for the current top element $\left(S_{k}\right.$, $\left.P_{k 1}, P_{k 2}\right), P_{k 1} \in R_{i}, P_{k 2} \in R_{j}$, if $R_{i}, R_{j}$ are different image regions and $\operatorname{Merging}\left(R_{i}, R_{j}\right)$ is true, merge the two regions. Then pop and read the next top element until $C$ is empty. The image will be finally partitioned into regions.

The proposed algorithm could be summarized as follow and the flow chart is shown in Fig. 4.

Step 1: Initialize the segmentation parameters which dominate the segmentation procedure and results

Step 2: Construct the neighbor pairwise pixel stack in increasing order of spectral difference according to Eqs. 7 and 8

Step 3: Read the current top element of stack and compute the heterogeneity between the two neighbor regions, hereby determine whether they can be merged

Step 4: Stop if the stack is empty. Otherwise pop and go back to Step 3 


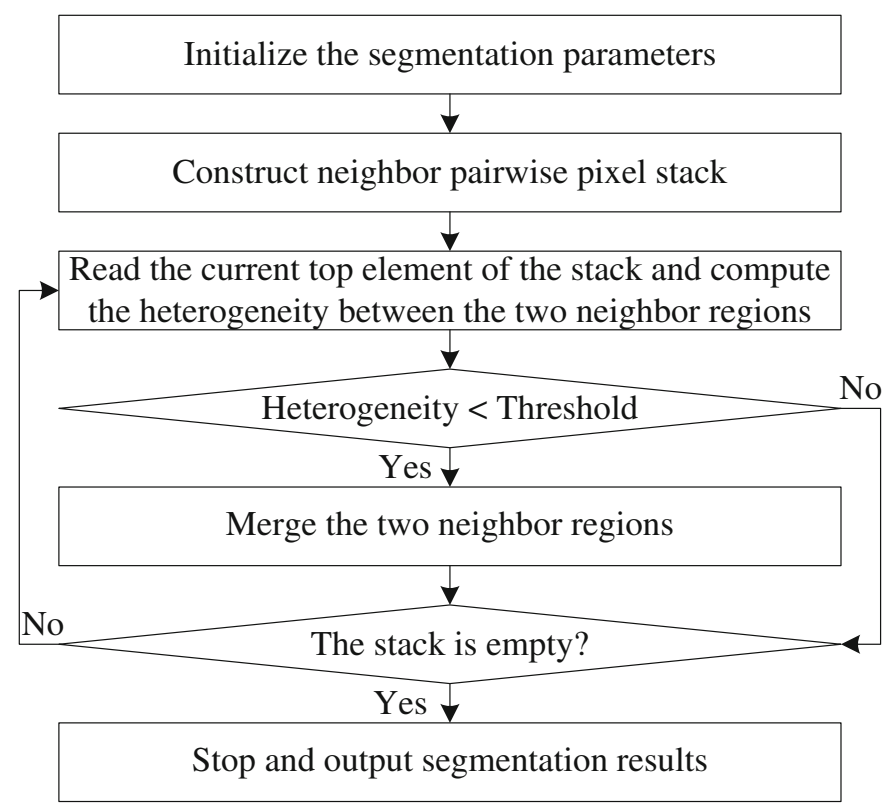

Fig. 4. Flow chart of the proposed algorithm

\subsection{Analysis of Time Complexity}

Suppose $H$ and $W$ are height and width of the given image respectively, the time complexity of the conventional region growing algorithm based on local mutual best fitting heuristics is $O\left((H W)^{2}\right)$ [7]. Instead of depending on any seed points, the proposed algorithm carries out region growing according to the merging criterion and traversal of the stack. The merge sort algorithm [28] is utilized for constructing neighbor pairwise pixel stack in the proposed algorithm. It is obvious that the time complexity of the proposed algorithm depends solely upon that of merge sort algorithm and the size of neighbor pairwise pixel stack. Consequently, the time complexity is reduced to $O\left(H W \log _{2}(H W)\right)$ in the proposed algorithm.

\section{Experimental Results and Analysis}

In order to test the effectiveness and adaptability of the proposed algorithm and verify the enhancement of segmentation efficiency based on the proposed algorithm, experimental results are given in this section. The region growing algorithm based on local mutual best fitting heuristics demonstrated in Sect. 2.1 is utilized as a contrastive algorithm and the segmentation accuracy is evaluated by Carleer evaluation method demonstrated in Sect. 2.2. It is worth noting that in order to derive more objective and accurate experimental results, the two segmentation algorithms utilize the same merging criterion demonstrated in Sect. 3.2 in our experiments. 


\subsection{Experimental Data}

Experiments are carried out on two data sets of ZiYuan-3 (ZY-3) remote sensing images. ZY-3 is China's first civilian high resolution cartographic satellite launched in Jan. 9, 2012. The ZY-3 images contain four spectral bands with a $5.8 \mathrm{~m}$ spatial resolution and a panchromatic band with a $2.1 \mathrm{~m}$ spatial resolution. It should be noticed that the data used in the experiments are pan-sharpened multi-spectral images. The two data sets shown in Fig. 5 are sub-regions of Guiyang China with size of $256 \times 256$ pixels. The first image was acquired on Mar. 31, 2012; the other was acquired on Mar. 30, 2013. Multi-spectral image pan-sharpening is conducted on ENVI 4.7.

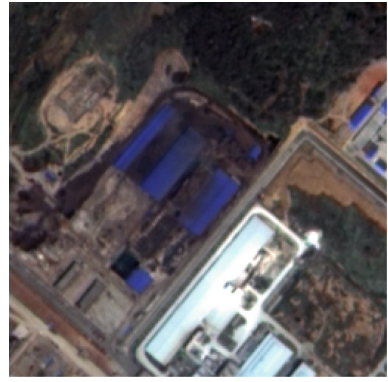

(a)

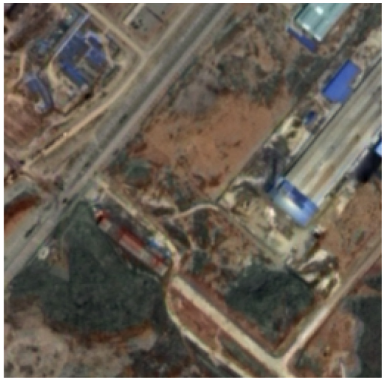

(b)

Fig. 5. Test images, (a) image $I_{1}$, (b) image $I_{2}$

\subsection{Relevant Statement}

For convenience, the proposed region growing algorithm, the conventional region growing algorithm based on local mutual best fitting heuristics, the percentage of mis-classified pixels and the ratio of region number are named as NSRG, TSRG, $E$ and $G$ respectively.

\subsection{Analysis of Image Segmentation Accuracy}

Owing to lack of abundant prior information about the experimental data, this paper does not utilize the segmentation results obtained by using specific segmentation parameters for evaluating segmentation accuracy; but instead based on a large number of preliminary experiments, we utilize quiet a few segmentation results for evaluating segmentation accuracy. The value of evaluation indices $E$ and $G$ in Carleer evaluation method can be firstly computed, and subsequently make a comparative analysis of $E$ while $G$ computed from NSRG is in consistency with that of TSRG. The detailed experimental results are as follows:

Firstly, for image $\mathrm{I}_{1}$, we found that when $G$ is less than 0.5 , under-segmentation is noticeable; when $G$ is greater than 6.0, over-segmentation is noticeable. Consequently, we choose the segmentation results in which $G$ is in the range $[0.5,6.0]$ for evaluating segmentation accuracy, as shown in Fig. 6a. 


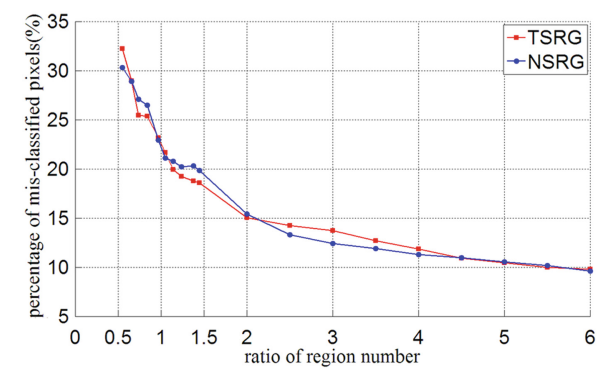

(a)

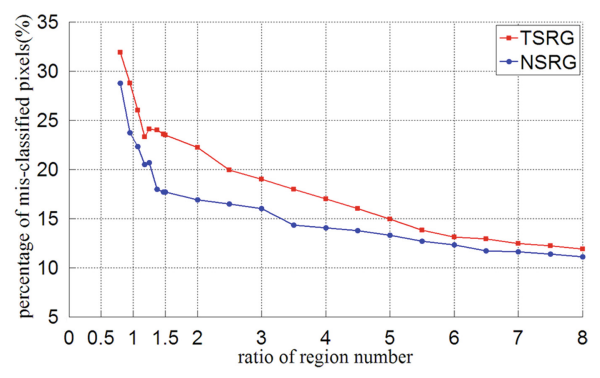

(b)

Fig. 6. Analysis of image segmentation accuracy based on two evaluation indices: $E$ and $G$, (a) for image $I_{1}$, (b) for image $I_{2}$

Secondly, for image $\mathrm{I}_{2}$, we found that when $G$ is less than 0.8 , under-segmentation is noticeable; when $G$ is greater than 8.0, over-segmentation is noticeable. Consequently, we choose the segmentation results in which $G$ is in the range $[0.8,8.0]$ for evaluating segmentation accuracy, as shown in Fig. $6 \mathrm{~b}$.

For the given reference segmentation, Carleer pointed out that $E$ will gradually reduce when $G$ is changed from low to high [3]; and this conclusion can be confirmed from Fig. 6. Further observation in Fig. 6, we can draw the following conclusions:

Firstly, for image $\mathrm{I}_{1}$, when $G$ is about $6.0, E$ computed from NSRG and TSRG are $9.60 \%$ and $9.77 \%$, respectively; when $G$ is reduced to $0.5, E$ computed from NSRG and TSRG have risen to $30.39 \%$ and $32.23 \%$, respectively; when $G$ is in the range $[0.5,6.0]$, on the premise of $G$ computed from NSRG is in consistency with that of TSRG, $E$ computed from NSRG and TSRG are roughly equal to each other.

Secondly, for image $\mathrm{I}_{2}$, when $G$ is about 8.0, $E$ computed from NSRG and TSRG are $11.10 \%$ and $11.91 \%$, respectively; when $G$ is reduced to $0.8, E$ computed from NSRG and TSRG have risen to $28.74 \%$ and $31.90 \%$, respectively; when $G$ is in the range $[0.8,8.0]$, on the premise of $G$ computed from NSRG is in consistency with that of TSRG, $E$ computed from NSRG is less than that of TSRG.

Thirdly, in general the shape of segmentation accuracy curves between $I_{1}$ and $I_{2}$ are similar to each other, but instead the segmentation accuracy of $I_{1}$ is slightly higher than that of $\mathrm{I}_{2}$ both segmented by NSRG and TSRG, this is the intrinsic expression of high resolution remote sensing images due to its complexity.

The aforementioned conclusions indicate that the segmentation accuracy of NSRG is not inferior to and even slightly higher than that of TSRG. Because NSRG algorithm carries out region growing according to the merging criterion (i.e. grow formula) and traversal of the neighbor pairwise pixel stack instead of depending on any seed points, consequently, each merging operation will be done from two neighbor regions in which the two neighbor pixels stored in the top of stack, while the spectral heterogeneity of these two neighbor pixels is smallest in the whole image at this moment. By applying NSRG algorithm to plenty of other high resolution remote sensing images, we can also derive satisfied segmentation results, and then a substantial foundation will be built for the subsequent remote sensing object detection or change detection. 
For images $I_{1}$ and $I_{2}$, to make a further comparative analysis of segmentation accuracy, take the segmentation results in which $G$ is equal to 1.76 for example, the reference segmentation and segmentation results by NSRG and TSRG algorithms are subsequently demonstrated in Figs. 7 and 8. Human eye is still a strong and experienced source for evaluation of image segmentation [15], compared with the reference segmentation by visual interpretation, we can draw the following conclusions:

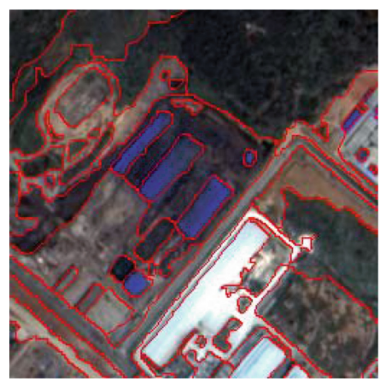

(a) reference segmentation

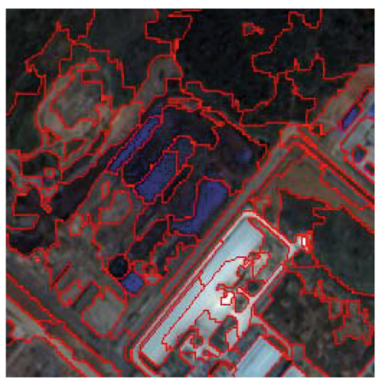

(b) result of NSRG algorithm, $E=16.84 \%, G=1.76$

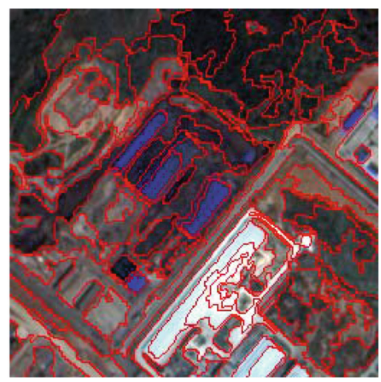

(c) result of TSRG algorithm, $E=18.05 \%, G=1.76$

Fig. 7. The segmentation results of image $I_{1}$ both by NSRG and TSRG algorithms

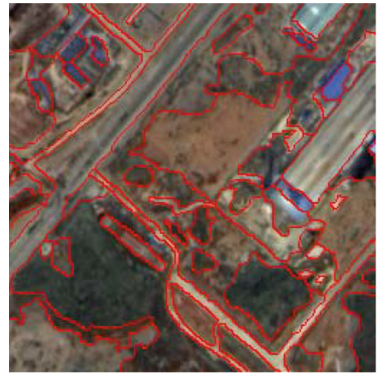

(a) reference segmentation

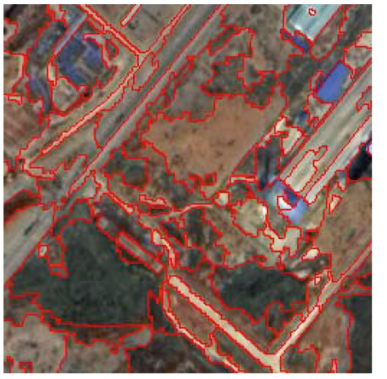

(b) result of NSRG algorithm, $E=17.56 \%, G=1.76$

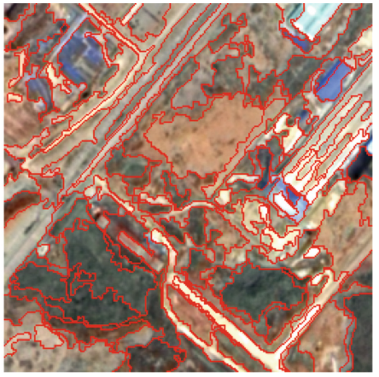

(c) result of TSRG algorithm, $E=21.73 \%, G=1.76$

Fig. 8. The segmentation results of image $I_{2}$ both by NSRG and TSRG algorithms

Firstly, there is no evident under-segmentation in segmentation results obtained by NSRG algorithm; some surface features comprise over-segmentation phenomena, but the primary surface features, such as roads, forest, bare land and houses were well segmented. Shortridge pointed out that a desirable segmentation result should comprise reasonable over-segmentation phenomena and no evident under-segmentation phenomena [29]; this indicates the segmentation results obtained by NSRG algorithm are reasonable.

Secondly, by contrast, the segmentation results obtained by NSRG algorithm are evident closer to respective reference segmentation and the overall segmentation 
accuracy is higher; the segmentation results obtained by TSRG algorithm are seem to be reduced to fragments, meanwhile the shape and boundary of image regions are more irregular.

Thirdly, TSRG and NSRG algorithms utilize different segmentation strategies, consequently even if the same merging criterion and segmentation parameters are used for both of them, the region growing procedure are not exactly the same. In addition, the segmentation results with insignificant holes are often obtained by the two algorithms, a post-processing step is indispensable.

\subsection{Analysis of Image Segmentation Efficiency}

Compared with TSRG algorithm, the primary design goal of NSRG algorithm is to enhance the image segmentation efficiency on the premise of without debasing the segmentation quality. NSRG and TSRG algorithms were implemented in MATLAB on a personal computer with $2 \mathrm{GHz}$ Intel Core2 Duo CPU and 2 GB RAM. We make a comparative analysis of average execution time between NSRG and TSRG while $G$ computed from them are equal to each other. They are separately run 20 times; the experimental results are shown in Tables 1 and 2 .

Table 1. The segmentation efficiency of image $I_{1}$ both by NSRG and TSRG algorithms

\begin{tabular}{l|l|l|l|l}
\hline \multirow{2}{*}{$G$} & \multicolumn{3}{ll}{ Segmentation efficiency } & \multicolumn{2}{l}{ NSRG algorithm } \\
\cline { 2 - 2 } & \multicolumn{2}{l}{ TSRG algorithm } & \multicolumn{2}{l}{} \\
\cline { 2 - 2 } & Execution time (s) & Average time (s) & Execution time (s) & Average time (s) \\
\hline 5.84 & 229.95 & 316.30 & 9.05 & 9.71 \\
\hline 4.90 & 241.84 & & 9.11 & \\
\hline 3.78 & 259.04 & & 9.26 & \\
\hline 2.53 & 315.55 & & 9.38 & \\
\hline 1.53 & 351.46 & & 9.86 & \\
\hline 0.57 & 499.97 & & 11.59 & \\
\hline
\end{tabular}

Table 2. The segmentation efficiency of image $I_{2}$ both by NSRG and TSRG algorithms

\begin{tabular}{|c|c|c|c|c|}
\hline \multirow[t]{3}{*}{$G$} & \multicolumn{4}{|c|}{ Segmentation efficiency } \\
\hline & \multicolumn{2}{|l|}{ TSRG algorithm } & \multicolumn{2}{|l|}{ NSRG algorithm } \\
\hline & Execution time (s) & Average time (s) & Execution time (s) & Average time (s) \\
\hline 5.82 & 228.14 & \multirow[t]{6}{*}{307.52} & 9.26 & \multirow[t]{6}{*}{9.67} \\
\hline 4.48 & 251.59 & & 9.31 & \\
\hline 3.52 & 258.01 & & 9.39 & \\
\hline 2.79 & 301.86 & & 9.44 & \\
\hline 1.70 & 337.26 & & 9.53 & \\
\hline 0.79 & 466.27 & & 11.07 & \\
\hline
\end{tabular}


From Tables 1 and 2, we can draw the following conclusions:

Firstly, for images $I_{1}$ and $I_{2}$, the execution time of NSRG and TSRG gradually increase when $G$ is changed from high to low. This is mainly due to the reason that the segmentation scale gradually increases when $G$ is changed from high to low, consequently more neighbor image regions can be merged and the execution time automatically increases.

Secondly, for images $I_{1}$ and $I_{2}$, compared with TSRG algorithm, the average segmentation efficiency of NSRG has increased 32.57 times and 31.80 times respectively. NSRG algorithm has an absolute advantage in segmentation efficiency, this is mainly due to the reason that NSRG algorithm does not depend on any seed points and does not utilize local mutual best fitting heuristics for guiding region merging.

As discussed in Sect. 3.3, if the size of given image is $H \times W$ pixels, the time complexity of TSRG and NSRG algorithms are $O\left((H W)^{2}\right), O\left(H W \log _{2}(H W)\right)$ respectively,this can also be proved from Tables 1 and 2 . In view of this, a substantial foundation will be built for large data bulk of high resolution remote sensing image segmentation and the subsequent remote sensing object detection or change detection.

\section{Conclusions}

This paper proposes a region growing algorithm for high resolution remote sensing image segmentation, NSRG. Compared with the conventional region growing algorithm based on local mutual best fitting heuristics, the proposed algorithm constructs neighbor pairwise pixel stack instead of depending on any seed points. It carries out region growing according to the merging criterion (i.e. grow formula) and traversal of the stack. The image segmentation capability comparison experimental results have demonstrated that the image segmentation efficiency is enhanced by the proposed algorithm on the premise of without debasing the segmentation quality. More experiments carried out on publically available remote sensing image data sets and comparison with other existing segmentation algorithms will be added in the future work.

\section{References}

1. Schiewe, J.: Segmentation of high-resolution remotely sensed data-concepts, applications and problems. Int. Arch. Photogrammetry Remote Sens. Spat. Inf. Sci. 34(4), 380-385 (2002)

2. Zhang, Y.J.: Evaluation and comparison of different segmentation algorithms. Pattern Recogn. Lett. 18, 963-974 (1997)

3. Carleer, A.P., Debeir, O., Wolff, E.: Assessment of very high spatial resolution satellite image segmentations. Photogrammetric Eng. Remote Sens. 71(11), 1285-1294 (2005)

4. Adams, R., Bischof, L.: Seeded region growing. IEEE Trans. Pattern Anal. Mach. Intell. 16 (6), 641-647 (1994)

5. Burnett, C., Blaschke, T.: A multi-scale segmentation/object relationship modeling methodology for landscape analysis. Ecol. Model. 168, 233-249 (2003)

6. Siebert, A.: Dynamic region growing. In: Vision Interface, vol. 97. Kelowna (1997) 
7. Chen, Z., Zhao, Z.M.: A multi-scale remote sensing image segmentation algorithm based on region growing. Comput. Eng. Appl. 41(35), 7-9 (2005)

8. Xu, Y.S., Fang, Z.L.: Improved segmentation of remote sensing images based on watershed algorithm. In: International Conference on Consumer Electronics, Communications and Networks, pp. 4136-4139 (2011)

9. Li, L.: Adaptive multi-scale segmentation of high resolution remote sensing images based on particle swarm optimization. Int. Conf. Intell. Hum. Mach. Syst. Cybern. 1, 151-154 (2013)

10. Fan, J., Yau, D.K., Elmagarmid, A.K., Aref, W.G.: Automatic image segmentation by integrating color-edge extraction and seeded region growing. IEEE Trans. Image Process. 10 (10), 1454-1466 (2001)

11. Cui, W., Guan, Z., Zhang, Z.: An improved region growing algorithm for image segmentation. Int. Conf. Comput. Sci. Softw. Eng. 6, 93-96 (2008)

12. Tang, J.: Color image segmentation algorithm based on region growing. Int. Conf. Comput. Eng. Technol. 6, V6-634-V6-637 (2010)

13. Preetha, M.M.S.J., Suresh, L.P., Bosco, M.J.: Image segmentation using seeded region growing. In: International Conference on Computing, Electronics and Electrical Technologies, pp. 576-583 (2012)

14. Mirghasemi, S., Rayudu, R., Zhang, M.: A new image segmentation algorithm based on modified seeded region growing and particle swarm optimization. In: International Conference on Image and Vision Computing, pp. 382-387, 2013

15. Baatz, M., Schape, A.: Multiresolution segmentation: an optimization approach for high quality multi-scale image segmentation. Angewandte Geographische InformationsVerarbeitung XII, pp. 12-23 (2000)

16. Benz, U.C., Hofmann, P., Willhauck, G., Lingenfelder, I., Heynen, M.: Multi-resolution, object-oriented fuzzy analysis of remote sensing data for GIS-ready information. ISPRS J. Photogrammetry Remote Sens. 58(3), 239-258 (2004)

17. Zhang, H., Fritts, J.E., Goldman, S.A.: Image segmentation evaluation: a survey of unsupervised methods. Comput. Vis. Image Underst. 110(2), 260-280 (2008)

18. Yang, L., Albregtsen, F., Lonnestad, T., Grottum, P.: A supervised approach to the evaluation of image segmentation methods. In: Hlaváč, V., Šára, R. (eds.) Computer Analysis of Images and Patterns. LNCS, vol. 970, pp. 759-765. Springer, Heidelberg (1995)

19. Chabrier, S., Laurent, H., Emile, B., Rosenberger, C., Marche, P.: A comparative study of supervised evaluation criteria for image segmentation. In: Proceedings of the European Signal Processing Conference, pp. 1143-1146 (2004)

20. Zhang, Y.: A survey on evaluation methods for image segmentation. Pattern Recogn. 29(8), 1335-1346 (1996)

21. Correia, P., Pereira, F.: Objective evaluation of relative segmentation quality. Int. Conf. Image Process. 1, 308-311 (2000)

22. Correia, P.L., Pereira, F.: Stand-alone objective segmentation quality evaluation. EURASIP J. Appl. Sig. Process. 1, 389-400 (2002)

23. Lee, S.U., Chung, S.Y., Park, R.H.: A comparative performance study of several global thresholding techniques for segmentation. Comput. Vis. Graph. Image Process. 52(2), 171190 (1990)

24. Lim, Y.W., Lee, S.U.: On the color image segmentation algorithm based on the thresholding and the fuzzy c-means techniques. Pattern Recogn. 23(9), 935-952 (1990)

25. Van Droogenbroeck, M., Barnich, O.: Design of statistical measures for the assessment of image segmentation schemes. In: Gagalowicz, A., Philips, W. (eds.) CAIP 2005. LNCS, vol. 3691, pp. 280-287. Springer, Heidelberg (2005) 
26. Ge, F., Wang, S., Liu, T.: Image-segmentation evaluation from the perspective of salient object extraction. IEEE Comput. Soc. Conf. Comput. Vis. Pattern Recogn. 1, 1146-1153 (2006)

27. Unnikrishnan, R., Pantofaru, C., Hebert, M.: A measure for objective evaluation of image segmentation algorithms. In: IEEE Computer Society Conference on Computer Vision and Pattern Recognition-Workshops, pp. 34-34 (2005)

28. Cole, R.: Parallel merge sort. SIAM J. Comput. 17(4), 770-785 (1988)

29. Shortridge, A.: Practical limits of Moran's autocorrelation index for raster class maps. Comput. Environ. Urban Syst. 31(3), 362-371 (2007) 